\title{
ATTACHING AND EFFACING PATHOGENS: THE EFFECTOR ABC OF IMMUNE SUBVERSION
}

\section{Short running title: Immune subversion by AE pathogens}

\section{ABSTRACT (118 words)}

The innate immune response resembles an essential barrier to bacterial infection. Many bacterial pathogens have, therefore, evolved mechanisms to evade from or subvert the host immune response in order to colonise, survive and multiply. The attaching and effacing (AE) pathogens enteropathogenic E. coli (EPEC), enterohaemorrhagic E. coli (EHEC) and Citrobacter rodentium are Gram-negative extracellular gastrointestinal pathogens. They use a Type-3 secretion system to inject effector proteins into the host cell to manipulate a variety of cellular processes. Over the last decade, considerable progress was made in identifying and characterising the effector proteins of $A E$ pathogens that are involved in the inhibition of innate immune signalling pathways, in determining their host cell targets and elucidating the mechanisms they employ.

KEYWORDS: AE pathogens, innate immune response, inflammatory response, inflammasome, apoptosis, necroptosis

\section{MAIN BODY OF TEXT}

\section{AE pathogens}

Enteropathogenic and enterohaemorrhagic Escherichia coli (EPEC and EHEC) are non-invasive human gastrointestinal pathogens that belong to the family of attaching and effacing (AE) pathogens. This family also includes the mouse-specific pathogen Citrobacter rodentium. The main hallmark of infection by this family of pathogens is the formation of ultrastructural features on the cell surface termed attaching and effacing (AE) lesions. Bacterial attachment is marked by the formation of actin-rich pedestals underneath the adherent bacteria followed by the loss of intestinal microvilli (effacement) in the vicinity of the attachment site. The bacterial Type-3 secretion system (T3SS), a needle-like structure through which bacterial effector proteins are translocated directly from the bacteria into the host cell cytoplasm, is 
essential for the formation of AE lesions. These effectors include the translocated intimin receptor (Tir), which, upon translocation, integrates into the plasma membrane to interact with the bacterial outer membrane protein intimin. This interaction ensures intimate attachment of the bacteria to the cell and leads to the initiation of cell signalling pathways that recruit actin to form pedestals [1]. The effector proteins EspF and Map have been ascribed a role in the effacement of microvilli [1].

The proteins necessary to build the T3SS, as well as the effector proteins required for AE lesion formation, are encoded on the locus of enterocyte effacement (LEE) pathogenicity island, which is conserved in all $A E$ pathogens. Genome sequencing of the EPEC lab strain E2348/69 [2] identified six additional pathogenicity islands that seem to have been acquired by horizontal gene transfer. These islands are surrounded by mobile elements (i.e. prophages or integrases). The genes encoded within these islands possess a lower GC content than is typical for $E$. coli genes [1]. Furthermore, the proteins encoded by these genes have common eukaryotic protein domains, suggesting that these proteins may play a role inside the host cells [1]. The sequencing of additional EPEC, EHEC and Citrobacter rodentium genomes revealed minor differences in the amount and copy number of individual effector proteins [3].

\section{The innate immune response to bacterial infection}

Innate immune responses provide potent defence mechanisms against invading pathogens. Membrane-spanning receptors, so-called pattern recognition receptors (PRR) recognise molecular structures produced by or associated with bacterial pathogens such as lipopolysaccharides (LPS) and flagellin. These bacterial factors commonly referred to as pathogen-associated molecular patterns (PAMPs). The binding of PAMPs to the PRRs induces receptor dimerisation followed by binding of adaptor proteins to the cytoplasmic tail of the receptor. This, in turn, leads to the activation of a variety of cytoplasmic protein kinases, which transmit their signals via protein modifications such as phosphorylation or ubiquitination. These signalling cascades ultimately result in the activation and nuclear translocation of transcription factors, activating the expression of cytokine and chemokine genes. Release of cytokines and chemokines from the cell attracts immune cells to the site of infection and promotes bacterial clearance. 


\section{Immune subversion by AE pathogens- early reports}

Initial studies showed that infection of epithelial cells with EPEC or EHEC led to an inhibition of inflammatory cytokine production and NF- $\kappa B$ activation in the initial stages of infection [4-6]. Infection with wildtype EPEC inhibited IL-8 secretion in response to TNF $\alpha, \mathrm{IL}-1 \beta$ and EHEC flagellin in a T3-dependent manner [6]. Wildtype EPEC infection further led to the inhibition of phosphorylation and, hence, activation of the mitogen-activated protein kinases (MAPKs) ERK and p38 [4]. The phosphorylation of p65 was also inhibited, interfering with its translocation into the nucleus and subsequent inhibition of pro-inflammatory cytokine and chemokine transcription [4]. This effect on phosphorylation also occurred in a T3-dependent manner, as it was observed in wildtype-infected cells but not in cells infected with a translocation-deficient mutant. The same study precluded the involvement of the proteins encoded on the LEE by assessing a deletion mutant unable to translocate ten effector proteins (EspG, Orf3, EspH, EspF, Map, Tir, EspZ, NleA, NleF and $\mathrm{NleH}$ ) [4]. Inhibition of NF-kB translocation into the nucleus was also observed in EHEC infected cells. Here, too, the process was determined to be T3-dependent. Additionally, infection with EHEC strains was shown to interfere with the binding of NF- $\kappa B$ subunits to the DNA [7].

Since these initial reports on the subversion of innate immune signalling by AE pathogens, the past decade has seen an immense increase in research activity in this field and an explosion of insight into the bacterial and cellular factors involved in these processes. AE pathogens were shown to encode a multitude of effector proteins that specifically target components of innate immune signalling at different levels in the pathways. The concerted action of these effectors prevents host cells from mounting an effective immune response [8-14]. Their functions will be reviewed here.

\section{INTERFERENCE WITH THE PRO-INFLAMMATORY RESPONSE}

\section{NF-KB and MAPK signalling}

The host inflammatory response is essential to clear invading pathogens from the body. Host cells sense the presence of pathogen-associated molecular patterns 
(PAMPs) such as lipopolysaccharide (LPS) or flagellin on the surface of bacteria via membrane-spanning receptor proteins. The receptors commonly associated with the inflammatory response to AE pathogens are the Toll-like receptors (TLRs) TLR4 and TLR5, the tumour-necrosis factor receptor (TNFR) and the IL-1 $\beta$ receptor (IL-1R) $[15,16]$. TLRs recruit adaptor proteins such as MyD88, IRAK and TRAF6 to their cytoplasmic tails, which induces signalling cascades that involve the kinases RIPK1 and TAK1 and well as TAB2/3, ultimately leading to the phosphorylation and activation of the Inhibitor of $\kappa B$ Kinase (IKK) complex. Signalling can also diverge at the level of TRAF6 and TAK and continue via the MAPK pathway by activating MKK3/6 and p38/JNK [17]. This subsequently results in the activation and nuclear translocation of the transcription factor activator protein-1 (AP-1). The TNF receptor, on the other hand, binds the adaptor proteins TRADD and TRAF2 to induce signalling via RIPK1 and the TAK-TAB2/3 complex, which in turn activates the IKK complex. Signalling from both TLR and TNFR pathways converge at the level of the IKK complex, which induces the phosphorylation, ubiquitination and subsequent

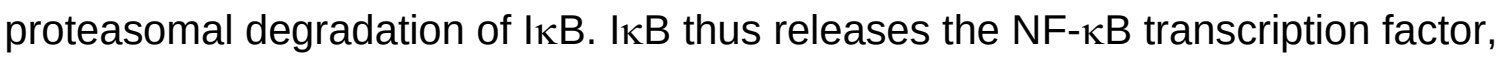
which migrates into the nucleus. Here, both AP-1 and NF-kB activate the transcription of pro-inflammatory cytokine genes $[15,16]$, which are subsequently released from the cell to attract immune cells to the site of infection.

Although initial results suggested that the effector proteins encoded on the LEE pathogenicity island do not play a role in the inhibition of the inflammatory response, newer studies have discovered at least one LEE effector with a minor role. As the functions of effectors in immune signalling are redundant, they can be easily overlooked.

The translocated intimin receptor (Tir) is encoded on the LEE and has long been considered the most crucial effector protein of AE pathogens, due to its significant role in bacterial attachment and pedestal formation. Transfection of Tir into mammalian cells inhibits NF-kB activation in response to TNF $\alpha$ stimulation via the TNFR and TLR4-receptor pathways by interacting with TNF $\alpha$ receptor-associated factor (TRAF) proteins such as TRAF2, resulting in their proteasome-independent degradation. This interferes with the activation of downstream signalling cascades and ultimately inhibits the NF-kB-mediated production of pro-inflammatory cytokines 
[18]. Tir was also shown to interact with the protein tyrosine phosphatases SHP-1 and SHP-2 via its two so-called immunoreceptor tyrosine-based inhibition motifs (ITIMs), $\mathrm{Y}_{490}$ and $\mathrm{Y}_{519}$, which leads to the de-ubiquitination of TRAF6 and to the inhibition of signalling via the IL-1 $\beta$ receptor $[19,20]$. Interaction with SHP-1 further facilitates the recruitment of SHP-1 to TAK1, inhibiting TAK1 phosphorylation. This, in turn, results in a reduction of TAK1 polyubiquitination and, hence, in the inhibition of downstream signalling [21]. An EHEC $\Delta$ tir mutant induced the release of a higher amount of pro-inflammatory cytokines in the intestine during infection of mice than wildtype EHEC, suggesting that Tir may play an important role in the inhibition of the pro-inflammatory response to infection in vivo [21]. However, while colonisation of wildtype EHEC and the $\triangle$ tir mutant were shown to be similar in this model, infection of mice with Shiga toxin-producing C. rodentium (C. rodentium $\phi s t x 2_{\text {dact }}$ ) and a respective $\Delta$ tir mutant resulted in a significant decrease of colonisation [22].

The first non-LEE effector proteins to be associated with inhibition of the proinflammatory response during infection were NleB and NleE. There are two functional copies of nleB in the genome of EPEC and EHEC, while only one, a homologue of NleB1 is found in Citrobacter rodentium. NleB2 is three amino acids shorter and shares $61 \%$ identity with NleB1, which is encoded on a different pathogenicity island. Interestingly, the pathogenicity island encoding NleB1 and NleE is the most conserved pathogenicity island apart from the LEE and nleB1 and nleE alleles are found in most isolates of EPEC, EHEC, and C. rodentium. Partly due to this, NleB1 is the more intensively studied of the two NleB homologues. The first studies suggested that NleB1 inhibits the pro-inflammatory response at the level of IKK or higher $[8,9,18]$. Interestingly, NleB1 only inhibits the TNFR but not signalling via the IL-1R pathway [9]. Gao et al. identified NleB1 as an Nglycosyltransferase which glycosylates GAPDH resulting in the inhibition of TRAF2 activation and downstream NF-кB signalling [23]. A DxD motif formed by amino acid residues $D_{221}$ and $D_{223}$ of NleB1 was identified as the protein's active site, and a mutant of NleB1 in which the aspartates are mutated is unable to glycosylate its targets [23-25].

EPEC and EHEC NleB1 as well as C. rodentium NleB block TNF $\alpha$-mediated NF-кB pathway activation. C. rodentium NleB and EHEC NleB1 bind to GAPDH and 
glycosylate it at two arginine residues $\left(\mathrm{R}_{197}\right.$ and $\left.\mathrm{R}_{200}\right)$ essential for GAPDH-mediated TRAF2 ubiquitination. Interestingly, while EPEC NleB1 also binds to GAPDH, only EHEC NleB1 glycosylates it, although the two proteins share $98 \%$ identity.

NleE, as NleB1, is highly conserved in AE pathogens [26]. It plays an essential role in the modulation of the pro-inflammatory response during infection, as it specifically blocks the translocation of activated NF- $\kappa B$ to the host cell nucleus by preventing the degradation of the inhibitor of kappaB $(I \kappa B)$. Blocking transcription factor translocation into the nucleus, in turn, leads to inhibition of the production and subsequent secretion of pro-inflammatory cytokines such as IL-8 [9]. NleE inhibits signalling via both, the TNFR and the IL-1R, in epithelial and dendritic cells [8-10], suggesting that the effector targets components shared by these pathways. NleE was shown to prevent IKK phosphorylation, and thus it was suggested that NleE blocks either TAK1 or IKK activation [8]. Another indication as to the target of NleE came from the observations that NleE inhibits NF-kB reporter activity driven by plasmid-expression of components belonging to the TNFR and IL-1R/TLR4 signalling pathways [18]. Here, NleE failed to inhibit luciferase activity driven by expression of TAK1 and IKK kinases, suggesting that it inhibits TAK1 function by either targeting TAK1 or factors needed for its activation [18].

In 2012, Zhang et al. identified NleE as a novel S-adenosyl-I-methioninedependent methyltransferase that inhibits NF- $\mathrm{KB}$ activation by methylating the zinccoordinating cysteine residue in the zinc finger domains of TAB2 and TAB3 [27]. This modification interferes with TAB2/3-TAK1 interaction and subsequent activation of TAK1, confirming the hypotheses of previous studies. Cysteine-methylated TAB2 and TAB3 lose the zinc ion necessary for their function, which diminishes their ubiquitin-chain binding activity [28].

A 6-amino acid motif, $\mathrm{I}_{209} \mathrm{DSYMK}_{214}$, at the C-terminus of NleE is critical for its immunosuppressive function [9]. Interestingly, both full-length NleE and NleE6A (a derivative in which each amino acid in the $\mathrm{I}_{209} \mathrm{DSYMK}_{214}$ motif was substituted with alanine) interact with TAB3 [29]. This suggests, as described for NleB above, the existence of a substrate recognition domain distinct from the domain required for activity [29]. Amino acids $\mathrm{G}_{49} \mid \mathrm{TR}_{52}$ of NleE were shown to be essential for its 
methylase activity as a respective mutant is unable to methylate TAB3 and consequently cannot inhibit IL-8 production [29].

While a $\triangle$ nleE mutant still shows residual inhibition of $\mathrm{I}_{\kappa} \mathrm{B}$ degradation, this can be eliminated upon further deletion of nleB [8] suggesting NleB may be necessary to enhance NleE activity [8]. The collaborative effect of NleB and NleE is further supported by the fact that nleE and nleB are encoded in one operon and that both are commonly found together in natural isolates of EPEC [17].

The effector proteins NleC and NleD are zinc-metalloproteases that are present in EPEC, EHEC and Citrobacter rodentium. They are encoded on the same pathogenicity island and share the conserved HExxH metalloprotease motif. They do, however, have different targets and are highly selective.

NleD, the lesser studied but no less interesting of the two zincmetalloproteases, is encoded just upstream of $\mathrm{NleC}$ in all AE pathogens. It confers EPEC with the ability to specifically cleave the MAP-kinases p38 and JNK but not ERK [12]. Baruch et al. showed that NleD cleaves JNK1 and JNK2 within their respective activation loops, which reduces the level of phosphorylated c-Jun [12], a subunit of the transcription factor AP-1. This, in turn, inhibits AP-1-mediated transcription of pro-inflammatory and pro-apoptotic genes [30]. The cleavage site in p38 was narrowed down to a region between amino acid residues $W_{187}$ and $M_{213}$ [30], which is close to the cleavage site published for JNK2, which corresponds to amino acid $\mathrm{P}_{182}$ in p38. It was further shown that NleD does not require any cellular cofactors for its activity [12].

Interestingly, a single amino acid, $\mathrm{R}_{203}$ is essential for mediating the cleavage of p38 but not JNK, suggesting a more stringent target specificity than previously thought [30]. Mutation of the residue to glutamate abolished cleavage and consequently AP-1-mediated reporter-gene activation.

$\mathrm{NleC}$, in contrast, exhibits its zinc-metalloprotease activity with high specificity for the transcription factor NF-кB subunit p65 [11-14]. However, while p65 certainly is the most intensely studied of its targets, NleC has been shown to cleave all five NF- $\kappa B$ subunits (p65, p50, p100, RelB, and c-Rel) [13, 31]. The cleavage of p65 was confirmed to depend on the catalytic activity of NleC as mutants with a disrupted 
catalytic site are unable to degrade p65 [11-14]. Cleavage occurs within the DNAbinding domain of the conserved Rel homology domain (RHD) between $\mathrm{C}_{38}$ and $\mathrm{E}_{39}$ of p65 [12], residues that are conserved in all NF-kB family members [32]. As for NleD, in vitro cleavage assays ruled out the need for cellular co-factors in the process $[12,32]$.

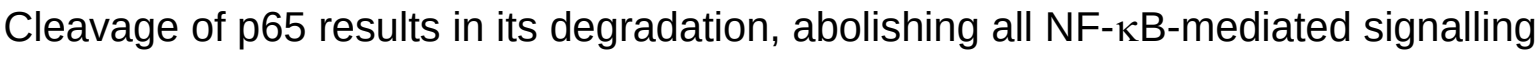
and inhibiting NF-kB-dependent pro-inflammatory cytokine production.

C. rodentium NleC also cleaves p65 between $\mathrm{C}_{38}$ and $\mathrm{E}_{39}$ during infection in

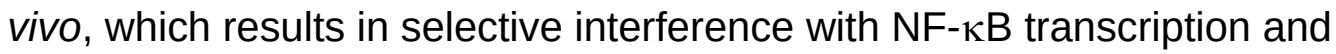
inflammation during infection of mice. Here, a C. rodentium mutant lacking NleC $(\Delta n l e C)$ increased the transcription of several pro-inflammatory cytokine genes including Cxcl1, Cxcl2, IIIb, Ifng and II22, and triggered an increased immune cell infiltration into the colon, when compared to wildtype C. rodentium infection [33].

A mutant of NleC, which lacks amino acids 1-65 ( $\left.\mathrm{NleC}_{66-330}\right)$, is unable to cleave p65 and inhibit NF-kB-mediated reporter-gene activation when transfected into host cells, while no effect was observed for a mutant missing residues 1-32, suggesting that residues 33-65 are required for NleC function [13]. Amino acids 266330 of NleC appear to be completely dispensable for the cleavage of p65 as their deletion has no effect, while removal of 30 additional amino acids (1-237) abolishes cleavage activity [13]. Analysing the crystal structure of NleC, residue $\mathrm{Y}_{227}$ was suggested to be involved in zinc-coordination within the protease active site [34, 35]. However, while certainly playing a role, the mutation of this residue does not entirely abolish the proteolytic activity of NleC [34].

Interestingly, mutation of $\mathrm{E}_{39}$ in p65 to alanine disrupts the ability of NleC to cleave p65 owing to the disappearance of a salt-bridge between $R_{232}$ of NleC and $E_{39}$ of p65. Two additional motifs in p65, $E_{22} I_{E_{25}}$ and $P_{177} V_{L} S_{180}$, are also crucial for recognition and binding of $\mathrm{p} 65$ by NleC. These motifs are located on adjacent, parallel strands of the p65 RHD [32].

The N-terminal cleavage product of p65 (p651-38) was shown to interfere with the interaction between native p65 and ribosomal protein S3 (RPS3), which confers promoter selectivity and transcriptional specificity for a subset of pro-inflammatory genes (e.g. IL-8) to the NF-кB transcription factor complex [36]. 
Further, NleC interacts with and degrades p300. P300 is an acetyltransferase that acts as a co-activator in the transcription of many host genes, including that of p65. The N-terminal TAZ1 domain of p300 is required for interaction with and degradation by NleC [37]. Overexpression of p300 decreases the ability of wildtype EPEC to dampen IL-8 secretion, while depletion of p300 decreases IL-8 secretion in cells infected with an EPEC $\Delta n l e C$ mutant [37].

NleC also reduces activation of the MAPK p38. While infection with $\Delta n l e C$ and wildtype $C$. rodentium results in similar colonisation, the $\Delta n l e C$ strain led to an increased colitis severity [33].

Lastly, NleC displays characteristics of bacterial AB toxins when added to the cells as purified protein. Recombinantly-expressed NleC can induce its uptake into the cell via endocytosis and follows the endosomal-lysosomal pathway. If taken up into the cell in this way, however, the activation of host cell signalling by cytokines such as IL-1 $\beta$ is required for NleC to cleave p65 [38].

Similar to nleB, there are two copies of $n l e H$ in the genome of EPEC and EHEC strains but only one copy in C. rodentium [3]. Although the two homologues of $\mathrm{NleH}$ in EHEC are 87\% identical [39] and both proteins are serine/threonine kinases with atypical kinase domains that require autophosphorylation to become active [4042], their functions are dissimilar [43].

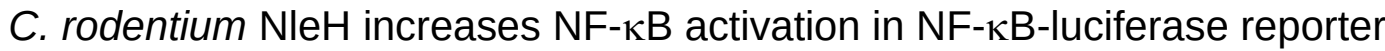
mice. Here, the levels of luciferase were higher in wildtype C. rodentium-infected mice than in mice infected with the $\Delta n l e H$ mutant. Wildtype-infected mice also expressed more TNF $\alpha$ compared to mice infected with $\Delta$ nleH [44]. Interestingly, following ectopic expression of either NleH from EHEC, only minor effects were observed in an NF-kB reporter assay [39], a result that is consistent with reports that NleH modulates NF-кB via the ribosomal protein S3 (RPS3).

EHEC and EPEC NleH effectors are functionally alike in their ability to inhibit the nuclear translocation of RPS3. Both $\mathrm{NleH}$ homologues bind the N-terminus of RPS3 with their respective N-termini, an interaction which is independent of the kinase function. While NleH1 reduces the nuclear abundance of RPS3 without altering p50 or p65 subunits or affecting the phosphorylation or subsequent degradation of $\mathrm{I}_{\kappa} \mathrm{Ba}, \mathrm{NleH} 2$ does not. NleH1 further represses the transcription of an 
RPS3-dependent NF-кB reporter but did not inhibit the transcription of RPS3independent reporters. In contrast, NleH2 activates RPS3-dependent reporters, as well as an AP-1-dependent reporter [43]. Furthermore, NleH1 suppresses ERK and p38 activation in vitro and in vivo while $\mathrm{NleH} 2$ suppresses only caspase-3 and p38 but not ERK [45].

The effector protein NleL of EHEC is a functional E3 ubiquitin ligase, and the cysteine residue $\mathrm{C}_{753}$ is essential for its activity [46]. NleL was shown to downregulate pedestal formation, and its $C$. rodentium homologue is required for efficient colonisation of the murine colon in vivo [46].

NleL uses its ubiquitin ligase activity to ubiquitinate the MAPK JNK, interfering with MKK $7 / J N K$ interaction and downstream signalling, which, in turn, suppresses AP-1 activation [47]. Interestingly, this inhibition of AP-1 was shown to affect AE lesion formation [47]. It may, however, also lead to the repression of AP-1-dependent transcription of pro-inflammatory and pro-apoptotic genes, although that aspect has not yet been investigated.

\section{Inflammasome signalling}

The inflammasome is a multimeric protein complex consisting of a sensor, an adaptor and Caspase-1. Upon sensing certain stimuli, the sensor recruits the adaptor protein ASC, which forms a bridge to inactive pro-Caspase-1. Incorporation of pro-Caspase-1 into the complex promotes complex oligomerisation and subsequently results in auto-proteolytic cleavage of pro-Caspase-1 to Caspase-1. Active Caspase-1, in turn, cleaves diverse substrates including the cytokines pro-IL$1 \beta$ and pro-IL-18 to their active forms. The NLRP3-inflammasome pathway requires a priming step which includes the sensing of PAMPs via TLRs to activate the NF- $\kappa B$ dependent transcription of NIrp3 and pro- $/ I 1 \mathrm{~b}$. This step is followed by activation of NLRP3/ASC/Caspase-1 complex assembly by, e.g. bacterial RNAs, pore-forming toxins, and T3SS-delivered bacterial proteins. NLRC4 stimulants, on the other hand, include components of T3SS itself. The NLRC4 protein contains a CARD domain, which allows it to recruit Caspase-1 without the necessity for ASC. The incorporation of ASC may, however, maximise the activity. NLR apoptosis inhibitory protein (NAIP), a co-factor of NLRC4 helps the complex to distinguish ligands. While there is 
only one NAIP protein in humans, four paralogues have been identified in mice, of which Naip1 shares the highest homology with human NAIP and recognises T3SS needle proteins. Naip2 senses T3SS rod proteins, and both Naip5 and Naip6 interact with bacterial flagellin. However, while the latter two have been shown to recognise flagellin from Salmonella spp, they do not associate with flagellins from EPEC or EHEC, which failed to activate the NLRC4 inflammasome. When infecting mice lacking NLRP3 and/or NLRC4 with C. rodentium, higher colonisation levels were observed, and the infection caused more severe colitis. Interestingly, while NLRP3 and NLRC4 played significant roles in the protection against $C$. rodentium early in infection, the inflammasome appears to be dispensable at late infection stages.

While several effector proteins of $A E$ pathogens interfere with the priming step of NLRP3 inflammasome signalling (i.e. with the NF- $\mathrm{KB}$ signalling pathway), NleA was shown to prevent IL-1 $\beta$ secretion independent of NF- $\kappa B$ signalling via the inhibition of Caspase-1 activation [48] and limits the number of inflammasome foci that form in response to LPS stimulation. NleA binds directly to NLRP3's pyrin and leucine-rich repeat domains and associates with both ubiquitinated and nonubiquitinated NLRP3, interrupting the de-ubiquitination of NLRP3, which is required for inflammasome activation [48].

The binding of the catalytic domain of Caspase- 4 by NleF, on the other hand, inhibits Caspase-4-mediated IL-18 processing during an EPEC infection [49]. Similarly, NleF-dependent inhibition of Caspase-11 (which is considered to be the murine homologue of human Caspase-4/-5) in colons of mice prevented the secretion of IL-18 and the influx of neutrophils during the early stages of $C$. rodentium infection [49].

\section{SUBVERSION OF CELL DEATH PATHWAYS}

\section{Apoptosis}

When inflammatory signalling is inhibited, TRADD dissociates from the TNF receptor and induces the recruitment of FADD and pro-Caspase-8 to form Complex Ila. This recruitment is followed by the activation of Caspase-8, which ultimately results in 
apoptotic cell death [50]. Alternatively, recognition of FAS ligand (FasL) by the FAS receptor results in the recruitment of FADD and pro-Caspase-8 to form a complex known as the death-inducing signalling complex (DISC). DISC formation also leads to apoptotic cell death through the activation of Caspase-8 [50].

While NleB was initially identified for its ability to inhibit the inflammatory response downstream of TNFR but not IL-1R, a different role emerged when new targets of its $\mathrm{N}$-glycosylating function were identified $[24,25]$. In 2013, the death domain-containing proteins TRADD, FADD and RIPK1 were shown to be glycosylated at a conserved arginine in their death domain (DD), a modification that inhibits the formation of the DISC complex and effectively shuts down apoptosis [24, 25]. Here, too, the DxD motif of NleB is essential for its function.

Closer investigation revealed that delivery of NleB during EPEC and C. rodentium infection caused a rapid, preferential and highly stable modification of $R_{117}$ in FADD $[51,52]$. Interestingly, the arginine residue is conserved in only one-third of all DD-containing proteins in humans, including FADD, TRADD, TNFR1, RIPK1 and FAS [51]. Upon infection, NleB modifies the death domains of TNFR1 and RIPK1 as well as FADD completely. A considerable portion of the FAS death domain is also glycosylated, while the DDs of MyD88 and IRAK1, which do not contain the conserved arginine, remain unaffected by NleB1 [51]. NleB1 derivatives in which either $E_{253}, Y_{219}$, or PILN ${ }_{63-66}$ were exchanged to alanine bound to, but did not glycosylate FADD. A further mutant, NleB1 PDG ${ }_{236-238} A A A$, did neither bind to nor glycosylate FADD.

In the murine infection model, deletion of $n l e B$ results in a significantly lower colonisation efficiency and increased colonisation clearance $[53,54]$. The effect of NleB on apoptosis is a significant contributor to this phenotype as a $\Delta n l e B$ mutant complemented with NleB colonised as well as wildtype C. rodentium, while complementation with the NleB DxD mutant had no such effect [24, 25]. In addition, C. rodentium $\Delta$ nleB strains complemented with either NleB $E_{253} A$ or NleB $Y_{219} A$ were also attenuated in mouse infections, indicating the importance of these residues in NleB virulence in vivo [55]. Furthermore, Fas-receptor knock-out $\left(I p r^{l^{-}}\right)$mice infected with wildtype $C$. rodentium reflected the $\triangle$ nleB mutants when tested, supporting the critical role for apoptosis during C. rodentium infection [24]. 
The cleavage of JNK by the zinc-metalloprotease NleD inhibits JNKdependent apoptosis in response to UV irradiation of infected cells. This phenotype is reduced in cells infected with an EPEC $\triangle n l e D$ mutant and confirmed by ectopically expressing NleD from a plasmid [12].

NleF was demonstrated to bind Caspases- $4,-8$ and -9 , resulting in the inhibition of the catalytic activity of these Caspases and the subsequent induction of apoptosis in vitro. The interference is mediated by the insertion of the NleF Cterminus into the Caspase active site as seen by co-crystallisation of the proteins [56]. Interestingly, although the C-terminus of $\mathrm{NleF}$ is involved in both processes, the interaction of the protein with Caspase- $4,-8$ and -9 is independent of its earlier described pro-inflammatory activity [57].

NleF prevents the proteolytic activity of Caspase-3, Caspase-8 and RIPK1 in response to FasR stimulation by FasL, ultimately blocking FasL-induced cell death. Interestingly, NleF did not contribute to the colonisation of mice in this study [58].

In addition to interfering with pro-inflammatory signalling, NleH effectors were also shown to block apoptosis [59]. They inhibit the elevation of cytosolic $\mathrm{Ca}^{2+}$ concentrations, nuclear condensation, Caspase-3 activation and membrane blebbing and promote cell survival [59]. During a $\mathrm{C}$. rodentium infection of mice, $\mathrm{NleH}$ inhibits pro-Caspase-3 cleavage at sites of bacterial attachment [59]. NleH binds within a 40 amino acid region in the N-terminus of Bax inhibitor-1 (BI-1). Knock-down of $\mathrm{BI}-1$, in turn, resulted in the loss of NleH's anti-apoptotic activity [59].

\section{Necroptosis}

Necroptosis or Caspase-independent cell death occurs when Caspase-8 is inhibited upon activation of cell death signalling. In this case, RIPK1 is de-ubiquitinated and subsequently phosphorylated. Phosphorylated RIPK1 then recruits RIPK3 through an interaction of their RHIM domains, leading to the phosphorylation of RIPK3 and its binding of the mixed-lineage kinase linker (MLKL) protein. MLKL is dualphosphorylated by RIPK3, oligomerises and translocates to the plasma membrane into which it is thought to insert, disrupting cell wall integrity [60]. 
Necroptotic cells show the morphological features of both, apoptosis and necroptosis, which include cell swelling and rupture of the cell membrane, resulting in the release of cytokines, ultimately culminating in inflammation of the affected tissue.

The ability of NleB to glycosylate the death domains of FADD, TRADD and RIPK1 was shown to block pro-inflammatory signalling via NF- $\kappa \mathrm{B}$ as well as apoptosis. Furthermore, as RIPK1 is an essential component of the necroptosis signalling pathway, NleB is also able to interfere with this type of cell death.

In addition, EspL, an effector protein encoded on the same pathogenicity island as NleB, was recently identified as a novel type of cysteine protease that cleaves the RHIM domain-containing proteins in their conserved RHIM domain, thereby also interfering with pro-inflammatory and cell death signalling [61]. Ectopic expression of EspL results in rapid cleavage of RIPK1, RIPK3, TRIF and ZBP/DAI and inhibition of TNF $\alpha$, LPS or poly(l:C)-induced (synthetic dsRNA sensed by TLR3) signalling and necroptosis. The amino acid residues $\mathrm{C}_{47} \mathrm{H}_{131} \mathrm{D}_{153}$ were identified as the catalytic site of the protein and mutation of either of these residues abolished protease function [61].

EspL was initially described to have an intermediate effect on the pathogenesis of C. rodentium in mice [53]. Recently, however, EspL's proteolytic activity was further shown to contribute to the persistent infection of mice by $C$. rodentium as the respective deletion mutant was cleared more rapidly than wildtype bacteria [61].

\section{Conclusion}

AE pathogens encode a vast repertoire of anti-inflammatory, anti-apoptotic and antinecroptotic effector proteins. Many of them have been acquired by horizontal gene transfer and are highly conserved among all AE pathogens, suggesting that the inhibition of the innate immune response is of great importance for the bacterial infection process.

Interestingly, infections with AE pathogens are generally considered to be inflammatory, and the pathology connected to these infections has, in large parts, 
been attributed to an active immune response. Both pro-and anti-inflammatory processes must, therefore, happen in the course of infection. While the inhibition of the immune response may be advantageous during the early stages of infection to allow the bacteria to attach and multiply, this necessity is lost at later time points.

\section{FUTURE PERSPECTIVE}

We now have a great deal of insight into the functional mechanisms effector proteins employ to interfere with the innate immune system during infection. The question remains, how this system works, interacts and cross-reacts in the intestine during infection. Pro-and anti-inflammatory and -apoptotic effectors are translocated into the cell by the bacterial T3SS. While we have an idea about the timing of effector translocation $[62,63]$, the timing of effector action is still mostly unknown. Better models for studying infections in vivo are continually being developed. To date, our knowledge is based on studies characterising the functions of single effector proteins by infection or transfection of cell lines and by assessing their in vivo relevance by using knock-out strains for mouse infections. New models will help us further our understanding of the disease process by studying the timing and collaboration of different factors during infection in vivo or by using sophisticated models, such as organoids, which more closely represent the native target milieu.

The influence of the intestinal microbiota in affecting bacterial infection, nutrient availability and access to metabolites have also been shown to affect the bacterial transcriptome and may, therefore, greatly influence the regulation of the T3SS and its effector proteins in vivo. There is a shift in the research on $A E$ pathogens towards addressing these issues as they are so essential to understanding the infection process in more detail.

The knowledge of effector protein functions and targets can also be used to aid the study of poorly understood cellular processes.

Additionally, the cellular processes affected by effector proteins may indicate as to possible targets for novel anti-virulence strategies.

Furthermore, the effector proteins themselves, especially those with a welldefined and limited target spectrum, can be used as or made into treatments. Particularly those effectors with anti-inflammatory functions can be developed into treatment options against certain inflammatory diseases. Several effector proteins 
from EPEC and other bacteria, such as Yersinia, have already been tested and provided some promising results [64] for the future.

\section{EXECUTIVE SUMMARY}

- The attaching and effacing pathogens EPEC, EHEC and C. rodentium are non-invasive gastrointestinal pathogens that use Type-3 secretion systems to inject effector proteins into host cells during infection.

- The innate immune response is essential for the control and clearance of bacterial pathogens.

- AE pathogens inhibit innate immune signalling pathways in a T3-dependent manner.

NF- $\kappa B$ and MAPK signalling

- Tir interferes with TRAF2 and TRAF6 activation by binding to SHP-1 and SHP-2, inhibiting TNFR-, TLR4- and IL-1R signalling

- NleB is a bacterial N-glycosyltransferase that modifies the death domains of FADD and RIPK1, resulting in the inhibition of TNFR signalling.

- NleC and NleD are zinc-metalloproteases. NleC cleaves the subunits of the NF- $\kappa \mathrm{B}$ transcription factor complex, while NleD targets the MAP kinases JNK and $\mathrm{p} 38$, resulting in degradation of the respective proteins.

- NleE functions as a cysteine-methyltransferase. It selectively methylates a conserved cysteine in TAB2 and TAB3, interfering with the activation of TAK1 and abolishing signalling via the TNF and IL-1 receptors.

- NleH binds and modifies RPS3, a specifier of NF- $\kappa B$, selectively inhibiting the transcription of RPS3-dependent cytokines.

Inflammasome signalling

- NleA binds to NLRP3, interfering with its de-ubiquitination and subsequent inflammasome activation

- NleF binds to Caspase-4, inhibiting the processing of pro-IL-18 to IL-18 
- The modification of the death domains of RIPK1, FADD, TRADD and others by NleB interferes with the recruitment of adapter proteins to the TNF and FAS receptors, abolishing Caspase-8 activation.

- NleD-mediated cleavage of JNK was shown to interfere with the pro-apoptotic signalling of JNK.

- NleF binds Caspases $-4,-8$ and -9 and was shown to prevent the activity of Caspase-3, Caspase-8 and RIPK1 in response to FAS signalling.

- NleH inhibits Caspase-3 activation and binds to Bax inhibitor-1.

Necroptosis

- NleB inhibits necroptosis by interfering with DISC complex formation and by modifying RIPK1.

- EspL is a cysteine protease, which cleaves the RHIM-domain containing proteins such as RIPK1 and RIPK3 within the conserved part of their RHIM domains.

\section{FIGURE LEGENDS}

Figure 1: Effector protein targets in the Inflammatory signalling pathway. Infection with $A E$ pathogens results in the activation of signalling via Toll-like receptors (TLRs) 4 and 5, the Interleukin-1 $\beta$ receptor (IL-1R) or the TNF receptor (TNFR), and initiation of diverse downstream signalling cascades. This ultimately result in the activation and nuclear translocation of the transcription factors and subsequent expression of cytokines and chemokines. Effector proteins (indicated in red) target several components of these signalling pathways, which leads to the inhibition of the host inflammatory response. The effector proteins display a number of different functions, such as initiating protein modifications, cleaving their target proteins or interference of target function by direct binding. Addition of an $\mathrm{N}$ acetylglucosamine ( $\mathrm{GlCNac})$, methylation $\left(\mathrm{CH}_{3}\right)$, ubiquitination $(\mathrm{Ub})$, and protein cleavage (scissors) are indicated where appropriate.

Figure 2: Inhibition of Inflammasome signalling by AE pathogens. After the priming step, which involves the recognition of PAMPs through pattern recognition 
receptors (PRR), the NF-kB signalling pathways is activated and results in the productions of pro-inflammatory cytokines pro-IL1 and pro-IL-18 as well as NLRP3. In the second step, Nod-like receptors NLRP3 and NLRC4 sense bacterial proteins or components and signalling ensues, which results in the formation of an inflammasome complex which includes pro-Caspase-1 which is cleaved to Caspase1. Caspase-1 can then cleave and activate IL-1 $\beta$ and IL-18. EPEC, EHEC and $C$. rodentium effector proteins (red) interfere with both, the priming step (described in Figure 1) and the formation of the NLRP3 inflammasome complex.

Figure 3: Interference with Death Receptor signalling by AE effector proteins. After activation of the TNF receptor (TNFR), the Fas receptor (CD95), the Death Receptor (DR) 3 or DR4/5, signalling cascades are activated which result in either apoptosis or, upon inhibition of caspase-8, in necroptosis. AE pathogen-translocated effector proteins (red) interrupt these signalling pathways to prevent host cell death. Every effector protein employs a unique mechanism including proteins modifications, target cleavage and direct interaction. Addition of an N-acetylglucosamine (GlcNac) and protein cleavage (scissors) are indicated where appropriate.

\section{TABLE LEGEND}

Table 1: List of effector proteins involved in the inhibition of innate immune signalling pathways, their targets and functions.

\section{REFERENCES}

1. Dean $\mathrm{P}$, Kenny $\mathrm{B}$. The effector repertoire of enteropathogenic $E$. coli: ganging up on the host cell. Current opinion in microbiology 12(1), 101-109 (2009).

2. Iguchi A, Thomson NR, Ogura $Y$ et al. Complete genome sequence and comparative genome analysis of enteropathogenic Escherichia coli O127:H6 strain E2348/69. J Bacteriol 191(1), 347-354 (2009).

3. Petty NK, Bulgin R, Crepin VF et al. The Citrobacter rodentium genome sequence reveals convergent evolution with human pathogenic Escherichia coli. J Bacteriol 192(2), 525-538 (2010).

4. Ruchaud-Sparagano MH, Maresca M, Kenny B. Enteropathogenic Escherichia coli (EPEC) inactivate innate immune responses prior to compromising epithelial barrier function. Cell Microbiol 9(8), 1909-1921 (2007). 
5. Nobe R, Nougayrede JP, Taieb F et al. Enterohaemorrhagic Escherichia coli serogroup O111 inhibits NF-(kappa)B-dependent innate responses in a manner independent of a type III secreted OspG orthologue. Microbiology 155(Pt 10), 3214-3225 (2009).

6. Sharma R, Tesfay S, Tomson FL, Kanteti RP, Viswanathan VK, Hecht G. Balance of bacterial pro- and anti-inflammatory mediators dictates net effect of enteropathogenic Escherichia coli on intestinal epithelial cells. Am J Physiol Gastrointest Liver Physiol 290(4), G685-694 (2006).

7. Hauf N, Chakraborty T. Suppression of NF-kappa B activation and proinflammatory cytokine expression by Shiga toxin-producing Escherichia coli. Journal of immunology (Baltimore, Md. : 1950) 170(4), 2074-2082 (2003).

8. Nadler C, Baruch K, Kobi S et al. The type III secretion effector NleE inhibits NFkappaB activation. PLoS pathogens 6(1), e1000743 (2010).

9. Newton HJ, Pearson JS, Badea L et al. The type III effectors NleE and NleB from enteropathogenic E. coli and OspZ from Shigella block nuclear translocation of NFkappaB p65. PLoS pathogens 6(5), e1000898 (2010).

10. Vossenkamper A, Macdonald TT, Marches O. Always one step ahead: How pathogenic bacteria use the type III secretion system to manipulate the intestinal mucosal immune system. Journal of inflammation 811 (2011).

11. Yen H, Ooka T, Iguchi A, Hayashi T, Sugimoto N, Tobe T. NleC, a type III secretion protease, compromises NF-kappaB activation by targeting p65/RelA. PLoS pathogens 6(12), e1001231 (2010).

12. Baruch K, Gur-Arie L, Nadler C et al. Metalloprotease type III effectors that specifically cleave JNK and NF-kappaB. The EMBO journal 30(1), 221-231 (2011).

13. Muhlen S, Ruchaud-Sparagano MH, Kenny B. Proteasome-independent degradation of canonical NFkappaB complex components by the NleC protein of pathogenic Escherichia coli. The Journal of biological chemistry 286(7), 5100-5107 (2011).

14. Pearson JS, Riedmaier P, Marches O, Frankel G, Hartland EL. A type III effector protease NleC from enteropathogenic Escherichia coli targets NF-kappaB for degradation. Molecular microbiology 80(1), 219-230 (2011).

15. Hayden MS, Ghosh S. Shared principles in NF-kappaB signaling. Cell 132(3), 344-362 (2008).

16. O'dea E, Hoffmann A. NF-kappaB signaling. Wiley interdisciplinary reviews. Systems biology and medicine 1(1), 107-115 (2009).

17. Pan ZK. Toll-like receptors and TLR-mediated signaling: more questions than answers. American journal of physiology. Lung cellular and molecular physiology 286(5), L918-920 (2004).

18. Ruchaud-Sparagano $M H$, Muhlen S, Dean P, Kenny B. The enteropathogenic E. coli (EPEC) Tir effector inhibits NF-kappaB activity by targeting TNFalpha receptorassociated factors. PLoS pathogens 7(12), e1002414 (2011).

19. Yan D, Quan H, Wang L et al. Enteropathogenic Escherichia coli Tir recruits cellular SHP-2 through ITIM motifs to suppress host immune response. Cellular signalling 25(9), 1887-1894 (2013).

20. Yan D, Wang X, Luo L, Cao X, Ge B. Inhibition of TLR signaling by a bacterial protein containing immunoreceptor tyrosine-based inhibitory motifs. Nature immunology 13(11), 1063-1071 (2012). 
21. Zhou R, Chen Z, Hao D et al. Enterohemorrhagic Escherichia coli Tir inhibits TAK1 activation and mediates immune evasion. Emerg Microbes Infect 8(1), 734-748 (2019).

22. Mallick EM, Mcbee ME, Vanguri VK et al. A novel murine infection model for Shiga toxin-producing Escherichia coli. J Clin Invest 122(11), 4012-4024 (2012).

23. Gao X, Wang X, Pham TH et al. NleB, a bacterial effector with glycosyltransferase activity, targets GAPDH function to inhibit NF-kappaB activation. Cell host \& microbe 13(1), 87-99 (2013).

24. Pearson JS, Giogha C, Ong SY et al. A type III effector antagonizes death receptor signalling during bacterial gut infection. Nature 501(7466), 247-251 (2013).

25. Li S, Zhang L, Yao Q et al. Pathogen blocks host death receptor signalling by arginine GlcNAcylation of death domains. Nature 501(7466), 242-246 (2013).

26. Zurawski DV, Mumy KL, Badea L et al. The NleE/OspZ family of effector proteins is required for polymorphonuclear transepithelial migration, a characteristic shared by enteropathogenic Escherichia coli and Shigella flexneri infections. Infect Immun 76(1), 369-379 (2008).

27. Zhang $L$, Ding $X$, Cui J et al. Cysteine methylation disrupts ubiquitin-chain sensing in NF-kappaB activation. Nature doi:nature10690 [pii]

10.1038/nature10690 (2011).

28. Zhang $L$, Ding $X$, Cui J et al. Cysteine methylation disrupts ubiquitin-chain sensing in NF-kappaB activation. Nature 481(7380), 204-208 (2011).

29. Zhang Y, Muhlen S, Oates CV, Pearson JS, Hartland EL. Identification of a Distinct Substrate-binding Domain in the Bacterial Cysteine Methyltransferase Effectors NleE and OspZ. The Journal of biological chemistry 291(38), 20149-20162 (2016).

30. Creuzburg K, Giogha C, Wong Fok Lung T et al. The Type III Effector NleD from Enteropathogenic Escherichia coli Differentiates between Host Substrates p38 and JNK. Infect Immun 85(2), (2017).

31. Jennings $E$, Esposito D, Rittinger K, Thurston TLM. Structure-function analyses of the bacterial zinc metalloprotease effector protein GtgA uncover key residues required for deactivating NF-kappaB. The Journal of biological chemistry 293(39), 1531615329 (2018).

32. Giogha C, Lung TW, Muhlen S, Pearson JS, Hartland EL. Substrate recognition by the zinc metalloprotease effector NleC from enteropathogenic Escherichia coli. Cell Microbiol 17(12), 1766-1778 (2015).

33. Sham HP, Shames SR, Croxen MA et al. Attaching and effacing bacterial effector NleC suppresses epithelial inflammatory responses by inhibiting NF-kappaB and p38 mitogen-activated protein kinase activation. Infect Immun 79(9), 3552-3562 (2011).

34. Li W, Liu Y, Sheng $X$ et al. Structure and mechanism of a type III secretion protease, NleC. Acta Crystallogr D Biol Crystallogr 70(Pt 1), 40-47 (2014).

35. Turco MM, Sousa MC. The structure and specificity of the type III secretion system effector NleC suggest a DNA mimicry mechanism of substrate recognition. Biochemistry 53(31), 5131-5139 (2014).

36. Hodgson A, Wier EM, Fu $\mathrm{K}$ et al. Metalloprotease NleC suppresses host NF-kappaB/inflammatory responses by cleaving p65 and interfering with the p65/RPS3 interaction. PLoS pathogens 11(3), e1004705 (2015). 
37. Shames SR, Bhavsar AP, Croxen MA et al. The pathogenic Escherichia coli type III secreted protease NleC degrades the host acetyltransferase p300. Cell Microbiol 13(10), 1542-1557 (2011).

38. Stolle AS, Norkowski S, Korner B et al. T3SS-Independent Uptake of the Short-Trip Toxin-Related Recombinant NleC Effector of Enteropathogenic Escherichia coli Leads to NF-kappaB p65 Cleavage. Front Cell Infect Microbiol 7119 (2017).

39. Holmes A, Lindestam Arlehamn CS, Wang D, Mitchell TJ, Evans TJ, Roe AJ. Expression and regulation of the Escherichia coli 0157:H7 effector proteins NleH1 and NleH2. PloS one 7(3), e33408 (2012).

40. Gao X, Wan F, Mateo $\mathrm{K}$ et al. Bacterial effector binding to ribosomal protein s3 subverts NF-kappaB function. PLoS pathogens 5(12), e1000708 (2009).

41. Grishin AM, Cherney $M$, Anderson DH, Phanse $S$, Babu M, Cygler M. NleH defines a new family of bacterial effector kinases. Structure 22(2), 250-259 (2014).

42. Halavaty AS, Anderson SM, Wawrzak Z et al. Type III effector NleH2 from Escherichia coli $0157: \mathrm{H} 7$ str. Sakai features an atypical protein kinase domain. Biochemistry 53(15), 2433-2435 (2014).

43. Pham TH, Gao X, Tsai K, Olsen R, Wan F, Hardwidge PR. Functional differences and interactions between the Escherichia coli type III secretion system effectors NleH1 and NleH2. Infect Immun 80(6), 2133-2140 (2012).

44. Hemrajani $\mathrm{C}$, Marches $\mathrm{O}$, Wiles $\mathrm{S}$ et al. Role of NleH, a type III secreted effector from attaching and effacing pathogens, in colonization of the bovine, ovine, and murine gut. Infect Immun 76(11), 4804-4813 (2008).

45. Kralicek SE, Nguyen M, Rhee KJ, Tapia R, Hecht G. EPEC NleH1 is significantly more effective in reversing colitis and reducing mortality than $\mathrm{NleH} 2$ via differential effects on host signaling pathways. Lab Invest 98(4), 477-488 (2018).

46. Piscatelli $\mathrm{H}$, Kotkar SA, Mcbee ME et al. The EHEC type III effector NleL is an E3 ubiquitin ligase that modulates pedestal formation. PloS one 6(4), e19331 (2011).

47. Sheng $X$, You $Q$, Zhu H et al. Bacterial effector NleL promotes enterohemorrhagic $E$. coli-induced attaching and effacing lesions by ubiquitylating and inactivating JNK. PLoS pathogens 13(7), e1006534 (2017).

48. Yen H, Sugimoto N, Tobe T. Enteropathogenic Escherichia coli Uses NleA to Inhibit NLRP3 Inflammasome Activation. PLoS pathogens 11(9), e1005121 (2015).

49. Pallett MA, Crepin VF, Serafini N et al. Bacterial virulence factor inhibits caspase-4/11 activation in intestinal epithelial cells. Mucosal Immunol 10(3), 602-612 (2017).

50. Giogha C, Lung TW, Pearson JS, Hartland EL. Inhibition of death receptor signaling by bacterial gut pathogens. Cytokine \& growth factor reviews 25(2), 235-243 (2014).

51. Scott NE, Giogha C, Pollock GL et al. The bacterial arginine glycosyltransferase effector NleB preferentially modifies Fas-associated death domain protein (FADD). The Journal of biological chemistry 292(42), 17337-17350 (2017).

52. El Qaidi S, Chen K, Halim A et al. NleB/SseK effectors from Citrobacter rodentium, Escherichia coli, and Salmonella enterica display distinct differences in host substrate specificity. The Journal of biological chemistry 292(27), 11423-11430 (2017).

53. Wickham ME, Lupp C, Mascarenhas $M$ et al. Bacterial genetic determinants of nonO157 STEC outbreaks and hemolytic-uremic syndrome after infection. J Infect Dis 194(6), 819-827 (2006). 
54. Kelly M, Hart E, Mundy R et al. Essential role of the type III secretion system effector $\mathrm{NleB}$ in colonization of mice by Citrobacter rodentium. Infect Immun 74(4), 23282337 (2006).

55. Wong Fok Lung T, Giogha C, Creuzburg K et al. Mutagenesis and Functional Analysis of the Bacterial Arginine Glycosyltransferase Effector NleB1 from Enteropathogenic Escherichia coli. Infect Immun 84(5), 1346-1360 (2016).

56. Blasche $\mathrm{S}$, Mortl M, Steuber $\mathrm{H}$ et al. The E. coli effector protein NleF is a caspase inhibitor. PloS one 8(3), e58937 (2013).

57. Pallett MA, Berger CN, Pearson JS, Hartland EL, Frankel G. The type III secretion effector NleF of enteropathogenic Escherichia coli activates NF-kappaB early during infection. Infect Immun 82(11), 4878-4888 (2014).

58. Pollock GL, Oates CVL, Giogha $C$ et al. Distinct Roles of the Antiapoptotic Effectors NleB and NleF from Enteropathogenic Escherichia coli. Infect Immun 85(4), (2017).

59. Hemrajani C, Berger CN, Robinson KS, Marches O, Mousnier A, Frankel G. NleH effectors interact with Bax inhibitor-1 to block apoptosis during enteropathogenic Escherichia coli infection. Proc Natl Acad Sci U S A 107(7), 3129-3134 (2010).

60. Sun L, Wang $\mathrm{H}$, Wang $\mathrm{Z}$ et al. Mixed lineage kinase domain-like protein mediates necrosis signaling downstream of RIP3 kinase. Cell 148(1-2), 213-227 (2012).

61. Pearson JS, Giogha C, Muhlen S et al. EspL is a bacterial cysteine protease effector that cleaves RHIM proteins to block necroptosis and inflammation. Nat Microbiol 2 16258 (2017).

62. Mills E, Baruch K, Aviv G, Nitzan M, Rosenshine I. Dynamics of the type III secretion system activity of enteropathogenic Escherichia coli. MBio 4(4), (2013).

63. Mills E, Baruch K, Charpentier X, Kobi S, Rosenshine I. Real-time analysis of effector translocation by the type III secretion system of enteropathogenic Escherichia coli. Cell host \& microbe 3(2), 104-113 (2008).

64. Ruter C, Hardwidge PR. 'Drugs from bugs': bacterial effector proteins as promising biological (immune-) therapeutics. FEMS Microbiol Lett 351(2), 126-132 (2014). 